\title{
A de novo STUB1 variant associated with an early adult-onset multisystemic ataxia phenotype
}

\author{
David Mengel ${ }^{1,2}$ (1) Andreas Traschütz ${ }^{1,2} \cdot$ Selina Reich ${ }^{1,2} \cdot$ Alejandra Leyva-Gutiérrez $^{1,2} \cdot$ Friedemann Bender $^{1,2}$. \\ Stefan Hauser ${ }^{1,2} \cdot$ Tobias B. Haack $^{3,4} \cdot$ Matthis Synofzik ${ }^{1,2}$
}

Received: 6 December 2020 / Revised: 3 March 2021 / Accepted: 15 March 2021 / Published online: 3 April 2021

(c) The Author(s) 2021

\begin{abstract}
Background Biallelic STUB1 variants are a well-established cause of autosomal-recessive early-onset multisystemic ataxia (SCAR16). Evidence for STUB1 variants causing autosomal-dominant ataxia (SCA48) so far largely relies on segregation data in larger families. Presenting the first de novo occurrence of a heterozygous STUB1 variant, we here present additional qualitative evidence for STUB1-disease as an autosomal-dominant disorder.

Methods Whole exome sequencing on an index patient with sporadic early-onset ataxia, followed by Sanger sequencing in all family members, was used to identify causative variants as well as to rule out alternative genetic hits and intronic STUB1 variants. STUB1 mRNA and protein levels in PBMCs in all family members were analysed using qRT-PCR and Western Blot. Results A previously unreported start-lost loss-of-function variant c. $3 \mathrm{G}>\mathrm{A}$ in the start codon of $S T U B 1$ was identified in the index case, occurring de novo and without evidence for a second (potentially missed) variant (e.g., intronic or copy number) in STUB1. The patient showed an early adult-onset multisystemic ataxia complicated by spastic gait disorder, distal myoclonus and cognitive dysfunction, thus closely mirroring the systems affected in autosomal-recessive STUB1-associated disease. In line with the predicted start-lost effect of the variant, functional investigations demonstrated markedly reduced STUB1 protein expression in PBMCs, whereas mRNA levels were intact.

Conclusion De novo occurrence of the loss-of-function STUB1 variant in our case with multisystemic ataxia provides a qualitatively additional line of evidence for STUB1-disease as an autosomal-dominant disorder, in which the same neurological systems are affected as in its autosomal-recessive counterpart. Moreover, this finding adds support for loss-of-function as a mechanism underlying autosomal-dominant STUB1-disease, thus mirroring its autosomal-recessive counterpart also in terms of the underlying mutational mechanism.
\end{abstract}

Keywords STUB1 $\cdot$ CHIP $\cdot$ Ataxia $\cdot$ Dominant $\cdot$ Early-onset ataxia $\cdot$ SCA48

Matthis Synofzik

matthis.synofzik@uni-tuebingen.de

1 Department of Neurodegenerative Diseases, Center for Neurology, Hertie Institute for Clinical Brain Research, University of Tübingen, Tübingen, Germany

2 German Centre for Neurodegenerative Diseases (DZNE), Tübingen, Germany

3 Institute of Medical Genetics and Applied Genomics, University of Tübingen, Tübingen, Germany

4 Centre for Rare Diseases, University of Tübingen, Tübingen, Germany

\section{Introduction}

While variants in STUB1 are well established to cause an autosomal-recessive early-onset multisystemic ataxia (SCAR16) [1-3], they have recently received also increasing support to be associated with autosomal-dominant ataxia (SCA48) [4-10]. Since the initial association of STUB1 with an autosomal-dominant form of spinocerebellar ataxia (SCA48) in 2018, 34 SCA48 patients originating from 17 families have been reported [5-12]. The complex clinical phenotype invariably included ataxia and, in most cases, cognitive and/or psychiatric dysfunction, variably associated with both hyperkinetic and hypokinetic movement disorders, pyramidal tract damage, and urinary symptoms. While segregation data in multigenerational families from previous 
studies provide plausible evidence in support of STUB1associated disorder as an autosomal-dominant ataxia disease, de novo occurrence of a heterozygous STUB1 variant in an ataxia patient would contribute a strong additional qualitative line of evidence.

Here, we report a case with a de novo heterozygous startlost c. $3 \mathrm{G}>\mathrm{A}$ mutation presenting with early adult-onset multisystemic ataxia complicated by cognitive dysfunction, distal myoclonus, and spastic gait disorder, thus providing qualitative novel additional evidence for STUB1-disease as an autosomal-dominant disorder. Moreover, biochemi$\mathrm{cal}$ analysis of the functional consequences of the $\mathrm{c} .3 \mathrm{G}>\mathrm{A}$ STUB1 start-lost mutation suggests loss-of-function ( $\mathrm{LoF})$ as an underlying mechanism in autosomal-dominant STUB1-disease.

\section{Methods}

\section{Patient recruitment, assessment, and whole exome sequencing}

The family ( 3 siblings, 2 parents, see Fig. $2 b$ ) and two unrelated controls were recruited as part of a continuous ongoing systematic deep-phenotyping and genotyping programme focussing on early-onset ataxias initiated by the Tübingen ataxia center, Hertie-Institute for Clinical Brain Research, Tübingen, Germany, since 2013. Standardized in-depth phenotyping (clinics, MRI), biosampling, and genetic work-up was performed in an index patient and, as available, his family members. A whole exome dataset with coverage on a diagnostic level (average coverage of the target region 17.6x, $96 \%$ covered $>20 \times$ ) from the index patient was investigated for rare variants in known and potentially novel disease genes with strict filter sets (minor allele frequency $<0.01 \%$ in gnomAD v2.1.1, Genome Aggregation Database, gnomad.broadinstitute.org; and combined annotation dependent depletion (CADD) score (phred-like, version 1.4) > 20 .

\section{Reagents and antibodies}

All chemicals and reagents were from Millipore-Sigma (Burlington, MA, USA) unless otherwise noted.

\section{PBMC isolation and culture}

For isolation of peripheral blood mononuclear cells (PBMCs), blood was collected into BD Mononuclear Cell Preparation Tubes (BD, Franklin Lakes, NJ, USA). Specimens were centrifuged at $1800 \times g$ for $20 \mathrm{~min}$ at room temperature (RT), the supernatant containing mononuclear cells was carefully aspirated, and cells washed using phosphate buffered saline (PBS). Residual erythrocytes were lyzed in lysis buffer ( $155 \mathrm{mM}$ ammonium chloride, $10 \mathrm{mM}$ potassium hydrogen carbonate, $0.1 \mathrm{mM}$ EDTA, $\mathrm{pH}$ 7.4), cells were washed again in PBS, and pelleted at $300 \times g$ for $10 \mathrm{~min}$ at RT. Finally, the cell pellet was resuspended in freezing medium (FCS (Thermo Fisher, Waltham, MA, USA) + 10\% DMSO) in cryotubes, and cells were frozen in liquid nitrogen until further use. PBMCs were thawed at $37{ }^{\circ} \mathrm{C}$, resuspended in RPMI media (Thermo Fisher, Waltham, MA, USA) containing 10\% FCS (Thermo Fisher, Waltham, MA, USA), and cultured for $48 \mathrm{~h}$ at $37^{\circ} \mathrm{C}$ and $5 \% \mathrm{CO}_{2}$.

\section{Generation of iPSCs and targeted STUB1 knockout with CRISPR/Cas9}

The generation of iPSCs from fibroblasts was performed according to a previously published protocol [13]. In brief, human dermal fibroblasts were nucleofected with $1 \mu \mathrm{g}$ of each episomal plasmid [pCXLE-hUL, pCXLE-hSK and pCXLE-hOCT4 (Addgene numbers 27080, 27078 and 27076, respectively)] with Nucleofector 2b (Lonza, Basel, Switzerland). Fibroblasts were cultivated in fibroblast medium before adding $2 \mathrm{ng} / \mathrm{mL}$ FGF2 (Peprotech, Hamburg, Germany) on day 2. Next day, medium was changed to Essential 8 (E8) medium with $100 \mu \mathrm{M}$ sodium butyrate. After 3-4 weeks, colonies were manually picked and expanded onto Matrigel-coated plates (Corning, NY, USA) in a feeder-free system in E8 medium. For generation of the STUB1 knockout line STUB1(-I-), exons 2 and 3 of STUB1 were targeted as previously reported [14].

\section{Neuronal differentiation of iPSCs}

iPSC-derived neurons were generated according to a published protocol [13]. In brief, iPSCs were seeded at a density of $3 \times 10^{5} / \mathrm{cm}^{2}$ in E8 medium supplemented with $10 \mu \mathrm{M}$ Y-27632. The following day, the medium was replaced by neural induction medium [1:1 N2/B27, $500 \mathrm{nM} \mathrm{LDN-}$ 193189 and $10 \mu \mathrm{M}$ SB431542], which, from there onwards, was changed every day. The neural induction medium was supplemented with $20 \mathrm{ng} / \mathrm{mL}$ FGF 2 on day 8 . The next day, cultures were split and seeded in N2/B27 medium with $20 \mathrm{ng} / \mathrm{mL}$ FGF2 and $10 \mu \mathrm{M} \mathrm{Y}-27632$ onto Matrigel-coated six-well plates. N2/B27 medium with $20 \mathrm{ng} / \mathrm{mL}$ FGF2 was added on the next day. From day 11 onward, cells were cultured in N2/B27 medium with a change of medium every other day. On day 26, cells were detached with Accutase and reseeded at $4 \times 10^{5} / \mathrm{cm}^{2}$ on poly-L-ornithine and Matrigelcoated wells. On day 27 and 29, the medium was changed to N2/B27 supplemented with $10 \mu \mathrm{M}$ PD0325901 (Tocris, Bristol, UK) and $10 \mu \mathrm{M}$ DAPT. From day 31 on cells were cultured in N2/B27 medium until day 36 on which cells harvested for protein isolation. 


\section{Protein isolation}

PBMCs and iPSC-derived neurons were lysed in RIPA buffer containing $1 \times$ complete protease inhibitor cocktail (Roche, Mannheim, Germany) for $30 \mathrm{~min}$ on ice, and vortexed every $10 \mathrm{~min}$ for $30 \mathrm{~s}$. Cell debris was pelleted at $20,000 \times \mathrm{g}$ for $15 \mathrm{~min}$ at $4{ }^{\circ} \mathrm{C}$. Protein concentration was determined using a BCA protein assay (Thermo Fisher Scientific, Waltham, MA, USA) according to the manufacturer's instructions.

\section{Western blot analysis}

Proteins were electrophoresed on hand-cast $10 \%$ Bis-Tris gels and transferred onto $0.45 \mu \mathrm{m}$ PVDF membrane (MerckMillipore, Burlington, MA, USA) at $100 \mathrm{~V}$ for $2 \mathrm{~h}$. Blots were incubated in tris-buffered saline containing $0.1 \%$ Tween-20 (TBS-T) and 5\% non-fat dry milk for $1 \mathrm{~h}$ at RT, and then incubated with primary antibody $(0.1 \mu \mathrm{g} / \mathrm{mL}$ antiSTUB1, ab134064, Abcam, Cambridge, UK) in $1 \times$ Roche Block (Roche Diagnostics, Mannheim, Germany) overnight at $4{ }^{\circ} \mathrm{C}$. Blots were then washed three times with TBS-T for $10 \mathrm{~min}$. Membranes were incubated with horseradish peroxidase-conjugated anti-rabbit antibodies (Jackson Immunosearch, Westgrove, PA, USA) for $1 \mathrm{~h}$ at RT, followed by $3 \times 10$ min washing with TBS-T. Immunoreactive bands were detected using ECL (Immobilon Western HRP Substrat, Merck-Millipore, Burlington, MA, USA) and visualized using the ChemiDOC MP Imaging System (Bio-Rad, Hercules, CA, USA). Next, blots were stripped with stripping buffer (0.025 M Glycine, $1 \%$ SDS, $1 \%$ Tween-20, pH 2.0) for $10 \mathrm{~min}$, washed $3 \times 10 \mathrm{~min}$ with TBS-T, and then reprobed with anti- $\beta$-actin monoclonal antibodies $(0.03 \mu \mathrm{g} /$ $\mathrm{mL}$ in $1 \times$ Roche Block, Merck-Millipore, Burlington, MA, USA) as loading control. Bands were quantified with ImageJ and normalized to the loading control.

\section{Quantitative real-time PCR}

Total mRNA was isolated from PBMCs using the High Pure RNA Isolation Kit (Roche Diagnostics, Mannheim, Germany) according to the manufacturer's protocol. Concentration and quality of RNA was determined by measuring absorbance at 260/280 nm with a NanoDrop ND1000 spectrophotometer (VWR, Radnor, PA, USA). A total of $200 \mathrm{ng}$ RNA was reverse-transcribed into cDNA using random hexamer primers and the Transcriptor High Fidelity cDNA Synthesis Kit (Roche Diagnostics, Mannheim, Germany) according to the manufacturer's instructions. For mRNA expression analysis, cDNA was diluted 1:10 and quantitative real-time PCR was performed on a Light Cycler 480 (Roche Diagnostics, Mannheim, Germany) using a touchdown PCR protocol. $1.5 \mu \mathrm{L}$ of cDNA dilution was mixed with $1.5 \mu \mathrm{L}$ of $20 \mu \mathrm{M}$ primer mix (forward: TCAAGGAGC
AGGGCAATCGT; reverse: CAGCGGGTTCCGGGTGAT ) and $7.5 \mu \mathrm{L}$ of LightCycler ${ }^{\circledR} 480$ SYBR Green I master mix (Roche Diagnostics, Mannheim, Germany). The specificity of PCR products was confirmed by melting curve analysis and efficiency was determined using standard curves. Housekeeping genes B2M, RNF111 and RNF10 were amplified to standardize the amount of sample cDNA. Analysis was performed with advanced relative quantification on the Light Cycler 480 Software 1.5.1.62 (Roche Diagnostics, Mannheim, Germany).

\section{Results}

A 34-year-old man from non-consanguineous German parents without family history of ataxia or dementia presented with progressive upper limb hyperkinetic movements starting at age 30 years, subtle and slowly progressive gait disturbance starting at age 33 years, and impaired fine motor skills starting age 34 years. Childhood development had been normal and after finishing high school, he had worked as a medical technician. Upon neurological examination, the patient showed-apart from cerebellar oculomotor disturbances (saccadic smooth pursuit eye movements, hypermetric saccades), truncal (ataxic stance and gait) and appendicular ataxia (limb dysmetria, kinetic tremor)—myoclonic jerks of the upper limbs, and spastic gait disturbance. Chorea, dystonia, parkinsonism, epilepsy, and urinary tract symptoms were absent. Clinical signs of neuropathy were not present, and secondary sexual characteristics were normal. In the consecutive 3 years from first examination, gait and stance became more unstable and upper limb ataxia worsened, as reflected by an increase of 2.5 points on the Scale for the Assessment and Rating of Ataxia (SARA: 15to 17.5 points) [15]. Neuropsychological evaluation including the Test battery for attentional performance (TAP), the Wechsler Memory ScaleRevised (WMS-R) and the Verbal learning and memory test (VLMT) indicated pronounced attention deficits and a decline in memory performance (age and education adjusted scores were lower or far below average). Visuospatial dysfunction, affective and behavioural symptoms were not observed, thus not meeting the full characteristics of the cerebellar cognitive affective syndrome [16], as described for other previous STUB1 patients [4, 6-8]. Due to cognitive decline and worsening ataxia, he had to change his workplace within the company and to reduce the workload to part-time. Prolongation of the central motor conduction time to the lower limbs provided corroborative electrophysiological evidence for pyramidal tract damage. Nerve conduction studies revealed-clinically inapparent-axonal motor neuropathy. In addition to severe cerebellar atrophy, MRI brain scan showed 
T2-weighted hyperintensity in the dentate nuclei and bilateral parietal atrophy (Fig. 1). Whole-exome sequencing of the index patient revealed a previously unreported heterozygous start-lost loss-of-function (LoF) variant c.3G $>\mathrm{A}$ in the start codon of STUB1 (NM_005861.4: p.?). This variant is absent in both 13,140 in-house WES/WGS datasets as well as in the 276,000 alleles of gnomAD. Not only all exonic, but also all intronic regions were covered with a coverage of $>20 \times$ (except merely a 56 base pair region in intron 1 , which was still covered with $>13 \times$ ) by whole exome sequencing, making it unlikely that a putative second variant in the intronic region of STUB1 (and thus a possible autosomal-recessive mode of inheritance) would have been missed (Fig. 2a). Likewise, WESbased CNV analysis by ClinCNV (https://www.biorxiv. org/content/10.1101/837971v1) did not reveal any CNVs within the chromosomal region $16 \mathrm{p} 13.3$. Together with the identification of two SNPs in neighbouring RHBDL1 in a heterozygous state (rs111852492 and rs370469600, located 3369 and 2851 bp upstream of the STUB1 transcript, respectively), these analyses did not provide any evidence that a putative CNV (e.g., a microdeletion) would
Fig. 1 Imaging findings of the index patient with the heterozygous de novo $S T U B 1$ variant. T2-weighted sagittal (left) and coronal (middle) and T1-weighted axial (right) MRI imaging showing severe cerebellar degeneration (blue arrows), atrophy of the parietal lobes (white arrows) and hyperintensity of both dentate nuclei (dark yellow arrows)
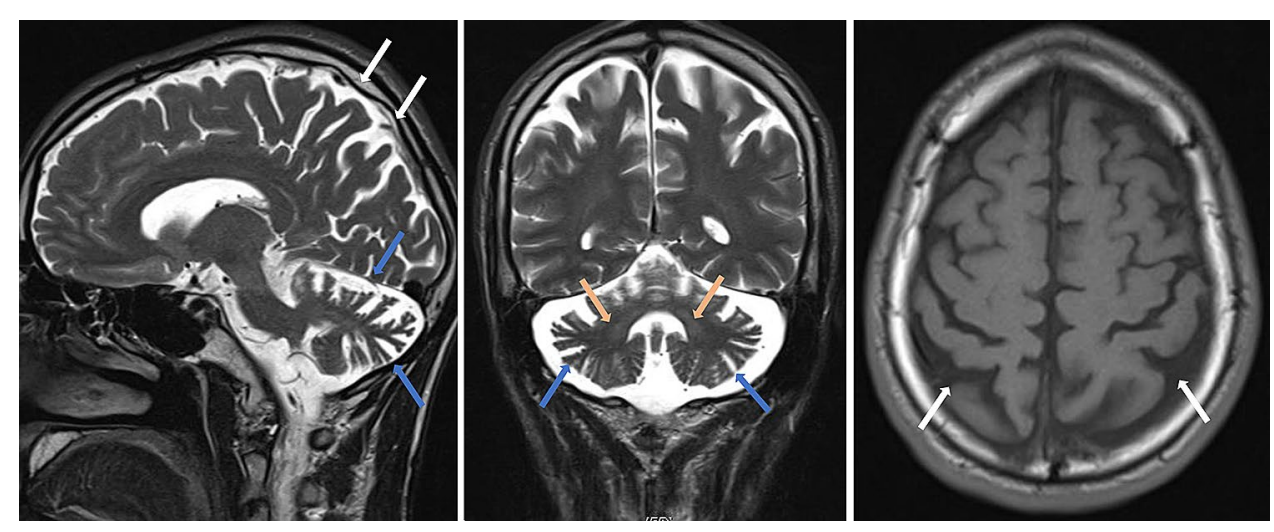
$>20 x \quad 13-19 x \quad>20 x$

a

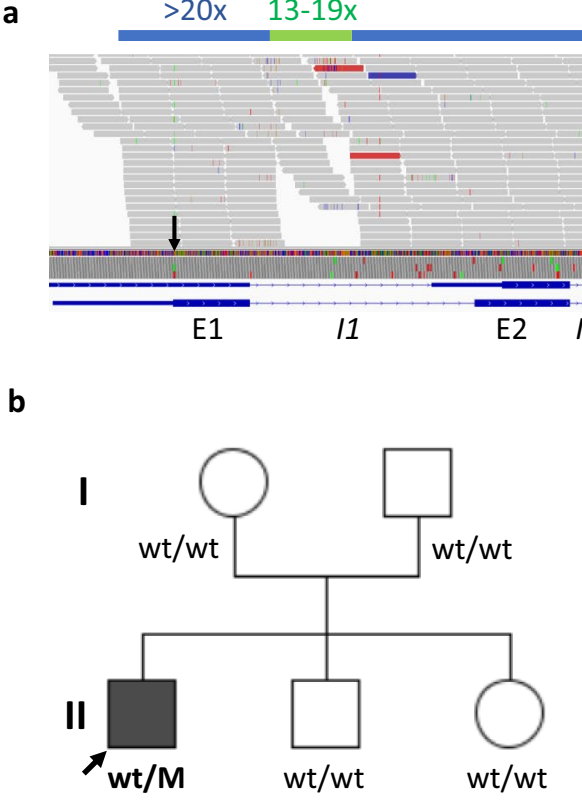

Fig. 2 Genetic findings of the index patient and his family. a WES of the index patient shows that all exonic (E1-E7) and intronic regions $(I 1-I 6)$ were covered with a coverage of $\geq 20 \times$ (except a 56 bp region in intron 1 , which was however, also still covered with $\geq 13 \times$, indicated in light green). The heterozygous c. $3 \mathrm{G}>\mathrm{A}$ $S T U B 1$ variant in the initiation codon is indicated with a black arrow.

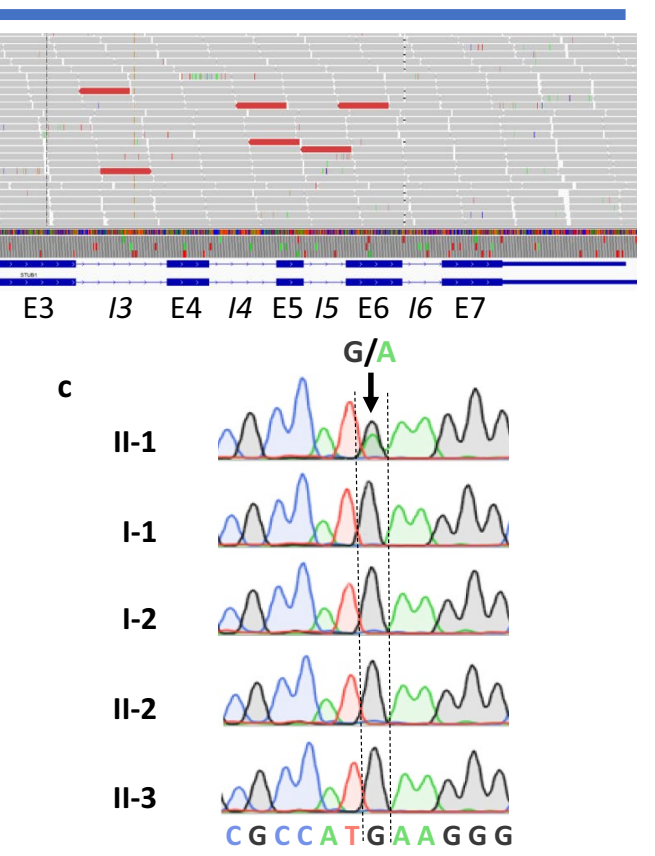

b Pedigree tree and c electropherograms demonstrating de novo occurrence of the heterozygous c. $3 \mathrm{G}>\mathrm{A}$ STUB1 variant $(=\mathrm{M})$ in the index patient. This mutation was absent in both unaffected parents and siblings. The filled symbol represents the affected patient, and open symbols asymptomatic family members. The index patient is marked with an arrow 
have been missed. Sanger sequencing confirmed presence of the variant in the index patient, and its absence in the other family members including both parents (Fig. 2b, c), with paternity confirmed by analysis of five short-tandemrepeat (STR) loci in the index patients and both parents, thus demonstrating de novo occurrence of this variant. The absence of the variant not only in the parents, but also in both siblings (Fig. 2b) not only corroborates potential pathogenicity of the variant, but also makes it less likely that a putative mosaicism in the parents might have been overlooked.

The c. $3 \mathrm{G}>\mathrm{A}$ variant was predicted in silico to cause a start-lost effect on protein translation. In line with this prediction, STUB1 protein expression in PBMCs was markedly decreased in the mutation carrier (reduced to $30 \%$ compared to his parents; Fig. 3a, b). Specificity of the antibody to detect STUB1 was demonstrated by use of cell lysates of STUB1 iPSC-derived neuronal cells where a strong band was visible at the predicted molecular weight of $35 \mathrm{kDa}$ for STUB1 wild-type, but not STUB1 knock-out cells, serving as positive and negative controls, respectively, for the Western Blot analyses. In sum, these protein analyses suggest loss-of-function (LoF) as the underlying disease mechanism of this $S T U B 1$ variant.

As predicted for a start-lost variant that only affects protein translation, not transcription, mRNA levels encoded by STUB1 were unchanged in PBMCs (Fig. 3c). Both STUB1 protein expression and mRNA levels were normal in the parents (Fig. 3a-c), adding further evidence that no putative mosaicism or second, inherited STUB1 variant (e.g., intronic or $\mathrm{CNV}$ ) in the family has been missed.

\section{Discussion}

With the first report of a de novo occurrence of a heterozygous STUB1 variant, our study adds a qualitative novel line of evidence that $S T U B 1$ variants, especially LoF variants, indeed cause also autosomal-dominant ataxia (SCA48). We functionally confirmed the predicted start-lost effect a

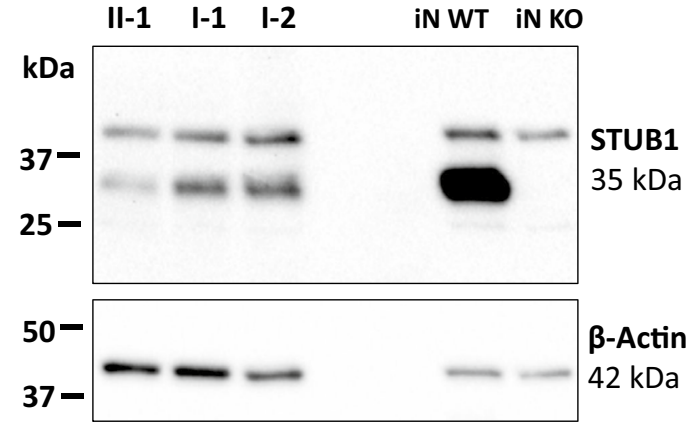

c

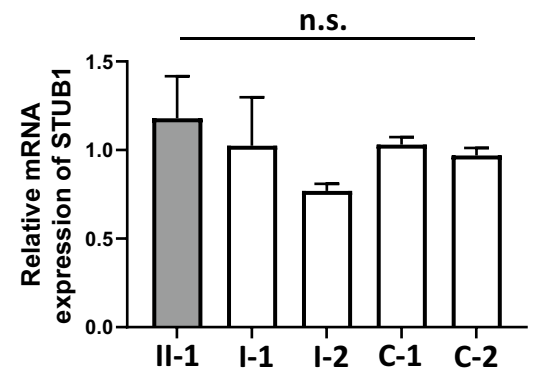

d b
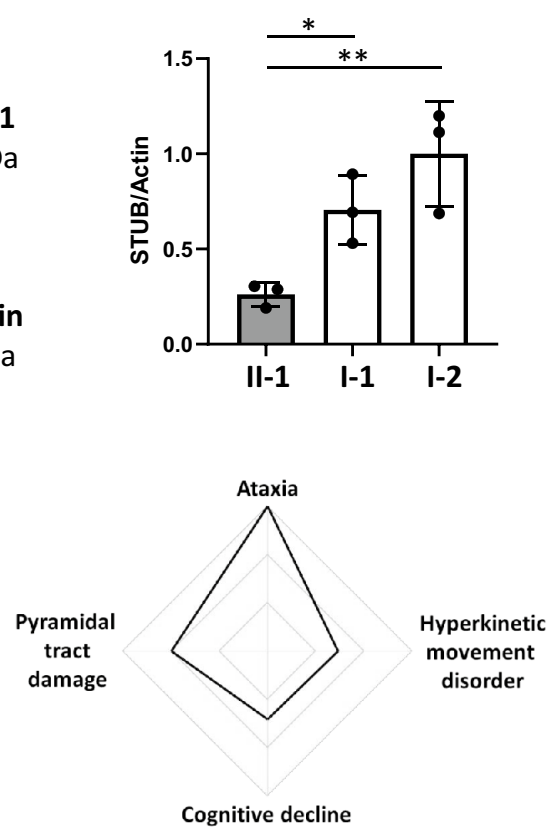

expression in the index patient relative to $\beta$-actin. $\mathbf{c}$ Expression of STUB1 mRNA was measured using qRT-PCR (in three independent experiments), and was similar in the index patient and his parents, and two unrelated controls (C-1 and C-2). Differences in means for b and $\mathbf{c}$ were assessed with ANOVA followed by Tukey's post-hoc test. Means and standard deviations are shown. n.s., non-significant $(p>0.05), * p<0.05, * * p<0.01$. d The phenotypic spectrum of the index patient with de novo STUB1-disease consists of ataxia, pyramidal tract damage, hyperkinetic movement disorder, and cognitive decline as part of the multisystemic disease spectrum in STUB1-disease 
of the variant, and ruled out other modes of inheritance other than de novo occurrence. Specifically, no evidence was found that this STUB1 variant was putatively inherited (and present e.g., just as a mosaic in one of the parents), with absence of the variant in all family members, including two siblings, and normal STUBI mRNA and protein expression levels in the parents. No evidence was found for a potential autosomal-recessive state of the variant, with a putative second intronic or structural STUB1 variant in the index patient ruled out by whole exome sequencing. This finding of de novo occurrence of STUB1 disease corroborates and extends current evidence of STUB1-disease as an autosomal-dominat disorder, which so far has relied mainly on segregation data in larger families and enrichment in mutational burden analyses $[4,5]$.

Moreover, our findings suggest loss-of-function (LoF) as an underlying mechanism in autosomal-dominant STUB1disease, at least for the STUB1 mutation investigated here. These findings nicely complement findings from heterozygous STUB1 -/+ mice, which suggested haploinsufficiency as a possible disease mechanism in murine autosomaldominant STUB1-disease [17]. Moreover, they mirror clinical and experimental studies from autosomal-recessive STUB1-disease, where LoF has also been suggested as a causative mechanism [5]. However, STUB1 gain-of-function and dominant negative effects have also been discussed for autosomal-dominant STUB1-disease [5, 7, 10]. Additional functional studies are warranted to better understand the mutational disease mechanisms underlying autosomaldominant STUB1-disease, ideally by a comparative parallel investigation of multiple STUB1 mutations of different variant types (e.g., missense, splice, stop), and from different protein domains.

Our findings thus not only provide corroborative genetic and preliminary mechanistic evidence for STUB1-disease as an autosomal-dominant disorder, but also add further phenotypic evidence for the similarity of autosomal-dominant STUB1 disease with its autosomal-recessive counterpart. Autosomal-recessive STUB1-disease has been described to cause an early-onset multisystemic ataxia including cerebellar ataxia, pyramidal tract damage, hyperkinetic movement disorders and cognitive decline as the main affected systems $[1,2]$. These systems-as well as the early-onset of the disease-are exactly all mirrored by the case presented here, which presented with cerebellar ataxia, distal myoclonus, spastic gait disorder and cognitive dysfunction (Fig. 3d), and with structural brain damage extending beyond the cerebellum to the cerebral cortex. The presence of bilateral signal alterations of the dentate nuclei along with cerebellar atrophy support the notion of a common neuroradiological feature in SCA48, recently referred to as "crab sign" due to its morphological appearance [18]. Although this striking MRI imaging sign is very rare in hereditary ataxias, systematic imaging studies are warranted to investigate whether it is really fully specific to STUB1-disease.

Taken together, our findings (1) add an additional qualitative line of evidence for the existence of autosomal-dominant STUB1-disease (hereby corroborating several recent studies $[6,9])$, (2) provide support for loss-of-function as a mechanism underlying autosomal-dominant STUB1-disease, thus mirroring its autosomal-recessive counterpart and (3) demonstrate that autosomal-dominant STUB1-disease affects the same neurological systems as its autosomal-recessive counterpart. This suggests, in sum, that both modes of inheritance can be associated with a similar STUB1 disease condition and possibly even similar mutational mechanism.

Acknowledgements We are grateful to the patient and his family.

Funding Open Access funding enabled and organized by Projekt DEAL. This work was supported, in part, by the Deutsche Forschungsgemeinschaft (DFG, German Research Foundation) No 441409627, as part of the PROSPAX consortium under the frame of EJP RD, the European Joint Programme on Rare Diseases, under the EJP RD COFUND-EJP N 825575 (to MS and SR) and grant 779257 "SolveRD" from the Horizon 2020 research and innovation programme (to MS). TH and MS are members of the European Reference Network for Rare Neurological Diseases-Project ID No 739510. DM received support through a Clinician Scientist Fellowship from the Medical Faculty of Tübingen University.

Availability of data and materials Not applicable.

\section{Declarations}

Conflicts of interest The authors declare that they have no conflict of interest.

Ethical standards The study was also approved by the Ethical Review Board Tübingen, as part of a general approval to study the clinical and genetic characteristics of degenerative ataxia patients $(\mathrm{Az}$ 598/2011BO1).

Informed consent Written informed consent for genetic analyses and functional experiments of the blood from the patient, his relatives, and both control patients were obtained.

Open Access This article is licensed under a Creative Commons Attribution 4.0 International License, which permits use, sharing, adaptation, distribution and reproduction in any medium or format, as long as you give appropriate credit to the original author(s) and the source, provide a link to the Creative Commons licence, and indicate if changes were made. The images or other third party material in this article are included in the article's Creative Commons licence, unless indicated otherwise in a credit line to the material. If material is not included in the article's Creative Commons licence and your intended use is not permitted by statutory regulation or exceeds the permitted use, you will need to obtain permission directly from the copyright holder. To view a copy of this licence, visit http://creativecommons.org/licenses/by/4.0/. 


\section{References}

1. Synofzik M, Schule R, Schulze M, Gburek-Augustat J, Schweizer R, Schirmacher A, Krageloh-Mann I, Gonzalez M, Young P, Zuchner S, Schols L, Bauer P (2014) Phenotype and frequency of STUB1 mutations: next-generation screenings in Caucasian ataxia and spastic paraplegia cohorts. Orphanet J Rare Dis 9:57. https://doi.org/10.1186/1750-1172-9-57

2. Hayer SN, Deconinck T, Bender B, Smets K, Zuchner S, Reich S, Schols L, Schule R, De Jonghe P, Baets J, Synofzik M (2017) STUB1/CHIP mutations cause Gordon Holmes syndrome as part of a widespread multisystemic neurodegeneration: evidence from four novel mutations. Orphanet J Rare Dis 12(1):31. https://doi. org/10.1186/s13023-017-0580-x

3. Shi CH, Schisler JC, Rubel CE, Tan S, Song B, McDonough H, Xu L, Portbury AL, Mao CY, True C, Wang RH, Wang QZ, Sun SL, Seminara SB, Patterson C, Xu YM (2014) Ataxia and hypogonadism caused by the loss of ubiquitin ligase activity of the $\mathrm{U}$ box protein CHIP. Hum Mol Genet 23(4):1013-1024. https://doi. org $/ 10.1093 / \mathrm{hmg} / \mathrm{ddt} 497$

4. Roux T, Barbier M, Papin M, Davoine CS, Sayah S, Coarelli G, Charles P, Marelli C, Parodi L, Tranchant C, Goizet C, Klebe S, Lohmann E, Van Maldergen L, van Broeckhoven C, Coutelier M, Tesson C, Stevanin G, Duyckaerts C, Brice A, Durr A (2020) Clinical, neuropathological, and genetic characterization of STUB1 variants in cerebellar ataxias: a frequent cause of predominant cognitive impairment. Genet Med. https://doi.org/10. 1038/s41436-020-0899-x

5. De Michele G, Galatolo D, Barghigiani M, Dello Iacovo D, Trovato R, Tessa A, Salvatore E, Filla A, Santorelli FM (2020) Spinocerebellar ataxia type 48: last but not least. Neurol Sci 41(9):2423-2432. https://doi.org/10.1007/s10072-020-04408-3

6. Lieto M, Riso V, Galatolo D, De Michele G, Rossi S, Barghigiani M, Cocozza S, Pontillo G, Trovato R, Sacca F, Salvatore E, Tessa A, Filla A, Santorelli FM, Silvestri G (2020) The complex phenotype of spinocerebellar ataxia type 48 in eight unrelated Italian families. Eur J Neurol 27(3):498-505. https://doi.org/10.1111/ene. 14094

7. Genis D, Ortega-Cubero S, San Nicolas H, Corral J, Gardenyes J, de Jorge L, Lopez E, Campos B, Lorenzo E, Tonda R, Beltran S, Negre M, Obon M, Beltran B, Fabregas L, Alemany B, Marquez F, Ramio-Torrenta L, Gich J, Volpini V, Pastor P (2018) Heterozygous STUB1 mutation causes familial ataxia with cognitive affective syndrome (SCA48). Neurology 91(21):e1988-e1998. https:// doi.org/10.1212/WNL.0000000000006550

8. Palvadeau R, Kaya-Gulec ZE, Simsir G, Vural A, Oztop-Cakmak O, Genc G, Aygun MS, Falay O, Basak AN, Ertan S (2020) Cerebellar cognitive-affective syndrome preceding ataxia associated with complex extrapyramidal features in a Turkish SCA48 family. Neurogenetics 21(1):51-58. https://doi.org/10.1007/ s10048-019-00595-0

9. Mol MO, van Rooij JGJ, Brusse E, Verkerk A, Melhem S, den Dunnen WFA, Rizzu P, Cupidi C, van Swieten JC, Donker Kaat L (2020) Clinical and pathologic phenotype of a large family with heterozygous STUB1 mutation. Neurol Genet 6(3):e417. https:// doi.org/10.1212/NXG.0000000000000417

10. Chen DH, Latimer C, Yagi M, Ndugga-Kabuye MK, Heigham E, Jayadev S, Meabon JS, Gomez CM, Keene CD, Cook DG, Raskind WH, Bird TD (2020) Heterozygous STUB1 missense variants cause ataxia, cognitive decline, and STUB1 mislocalization. Neurol Genet 6(2):1-13. https://doi.org/10.1212/NXG.00000 00000000397

11. De Michele G, Lieto M, Galatolo D, Salvatore E, Cocozza S, Barghigiani M, Tessa A, Baldacci J, Pappata S, Filla A, Santorelli FM (2019) Spinocerebellar ataxia 48 presenting with ataxia associated with cognitive, psychiatric, and extrapyramidal features: a report of two Italian families. Parkinsonism Relat Disord 65:91-96. https://doi.org/10.1016/j.parkreldis.2019.05.001

12. Ravel JM, Benkirane M, Calmels N, Marelli C, Ory-Magne F, Ewenczyk C, Halleb Y, Tison F, Lecocq C, Pische G, Casenave P, Chaussenot A, Frismand S, Tyvaert L, Larrieu L, Pointaux M, Drouot N, Bossenmeyer-Pourie C, Oussalah A, Gueant JL, Leheup B, Bonnet C, Anheim M, Tranchant C, Lambert L, Chelly J, Koenig M, Renaud M (2021) Expanding the clinical spectrum of STIP1 homology and U-box containing protein 1-associated ataxia. J Neurol. https://doi.org/10.1007/s00415-020-10348-X

13. Schuster S, Heuten E, Velic A, Admard J, Synofzik M, Ossowski S, Macek B, Hauser S, Schols L (2020) CHIP mutations affect the heat shock response differently in human fibroblasts and iPSCderived neurons. Dis Model Mech. https://doi.org/10.1242/dmm. 045096

14. Schuster S, Saravanakumar S, Schols L, Hauser S (2019) Generation of a homozygous CRISPR/Cas9-mediated knockout human iPSC line for the STUB1 locus. Stem Cell Res 34:101378. https:// doi.org/10.1016/j.scr.2018.101378

15. Schmitz-Hubsch T, du Montcel ST, Baliko L, Berciano J, Boesch S, Depondt C, Giunti P, Globas C, Infante J, Kang JS, Kremer B, Mariotti C, Melegh B, Pandolfo M, Rakowicz M, Ribai P, Rola R, Schols L, Szymanski S, van de Warrenburg BP, Durr A, Klockgether T, Fancellu R (2006) Scale for the assessment and rating of ataxia: development of a new clinical scale. Neurology 66(11):1717-1720. https://doi.org/10.1212/01.wnl.0000219042. 60538.92

16. Schmahmann JD, Sherman JC (1998) The cerebellar cognitive affective syndrome. Brain 121(Pt 4):561-579. https://doi.org/10. 1093/brain/121.4.561

17. McLaughlin B, Buendia MA, Saborido TP, Palubinsky AM, Stankowski JN, Stanwood GD (2012) Haploinsufficiency of the E3 ubiquitin ligase C-terminus of heat shock cognate 70 interacting protein (CHIP) produces specific behavioral impairments. PLoS ONE 7(5):e36340. https://doi.org/10.1371/journal.pone. 0036340

18. Cocozza S, Pontillo G, De Michele G, Perillo T, Guerriero E, Ugga L, Salvatore E, Galatolo D, Riso V, Sacca F, Quarantelli M, Brunetti A (2020) The "crab sign": an imaging feature of spinocerebellar ataxia type 48. Neuroradiology 62(9):1095-1103. https://doi.org/10.1007/s00234-020-02427-7 